\title{
Towards Sub-cellular Modeling with Delaunay Triangulation
}

\author{
G. Grise * and M. Meyer-Hermann \\ Frankfurt Institute for Advanced Studies, Goethe University \\ Ruth-Moufang Strasse 1, 60438 Frankfurt, Germany
}

\begin{abstract}
In this article a novel model framework to simulate cells and their internal structure is described. The model is agent-based and suitable to simulate single cells with a detailed internal structure as well as multi-cellular compounds. Cells are simulated as a set of many interacting particles, with neighborhood relations defined via a Delaunay triangulation. The interacting subparticles of a cell can assume specific roles - i.e., membrane sub-particle, internal sub-particle, organelles, etc -, distinguished by specific interaction potentials and, eventually, also by the use of modified interaction criteria. For example, membrane sub-particles may interact only on a twodimensional surface embedded on three-dimensional space, described via a restricted Delaunay triangulation. The model can be used not only to study cell shape and movement, but also has the potential to investigate the coupling between internal space-resolved movement of molecules and determined cell behaviors.
\end{abstract}

Key words: cell shape, cell movement, sub-cellular model, delaunay triangulation, voronoi tessellation, surface reconstruction

AMS subject classification: 51P05, 7008, 68U05, 92B99

\section{Introduction}

The recent development of advanced experimental techniques in cell tracking (specially with the advent of two-photon imaging [1, 2, 3]) opened up great opportunities for theoretical biologists interested in modeling cell shape and migration. With these techniques the shape and movement of individual cells can be tracked inside living animals, generating data supposed to mirror the

\footnotetext{
${ }^{*}$ Corresponding author. E-mail: grise@ fias.uni-frankfurt.de
} 
real behavior of cells in vivo. Also, experimental techniques able to identify up to hundreds of molecules inside a cell at a determined time point emerged $[4,5]$, calling for a cell model on which molecules can be followed in a space-resolved manner.

Traditional methods like differential equations are only adequate to describe big systems, where the behavior of one individual entity alone is not relevant to the systems' overall outcome, and where the properties one is interested in can be averaged. In small systems, where particles must be individually identified and their behaviors independently studied, agent-based models are needed.

The migration of cells is intrinsically connected to their shape [6]. Therefore, a theoretical model aiming in describing cell migration has to take cell shape in consideration, which in turn requires knowledge about the internal structure of the cell. Cells, in this case, cannot be simulated as a single point in space, but more as a set of sub-particles interacting according to given properties. At the same time, a model intending to follow molecules inside a cell requires the same properties: it must treat cells individually and it needs an internal resolution of the cell.

In this article a novel model framework to describe cell shape and behavior will be explained. This method describes a cell as a set of many interacting sub-particles, and it might be suitable to simulate both cell shape derived from two-photon imaging data [7, 8] and the tracking of molecules inside a cell as obtained from toponome experiments $[4,5]$. The method is suitable for the simulation of single cells as well as multi-cellular systems.

The structure of the article is the following: in Section 2. the available methods to simulate cell shape involving subcellular structure will be discussed and compared, and the model introduced. In Section 3. the mathematical background and technical details needed for the implementation of the model are presented. In Section 4. the different sub-particles are introduced, as well as the potentials and the integration algorithm used. The method to define the neighborhood relations between the membrane sub-particles is also explained. Finally, Section 5. clarifies the process used to generate initial configurations to be used within the framework.

\section{Sub-cellular models}

There exist a few theoretical models aiming at describing cell shape and migration. The most traditional one is the Potts model [9], a generalization of the Ising model with multiple spin states. The space is discretized as a lattice, with each volume element having a spin associated to it. A cell is then defined as all volume elements with the same spin state. Changes in shape and, therefore, the movement of cells are usually governed by thermodynamic interactions. The Potts model is very widespread in biological simulations. The model uses a lattice, which means cells and space are not smooth but discretized. A change of the cells' shape is always correlated to a change of the cells volume, since a subunit on the lattice has to change its spin state to the one of its neighbor, if the cell is to move. These unphysical changes in cell volume associated with cell movement are negligible in the limit of many subcellular nodes per cell.

An alternative model architecture which intrinsically includes the one- and the multi-subunit limit was also developed [10]. In the model, called hyphasma, all reaction kinetics are formulated 
as reaction rates and actions are taken accordingly to probabilistic decisions. The movement of the cells is formulated in terms of forces acting on the subunits of the cells.

Off-lattice models, widely used in the simulation of multi-cellular systems [11, 12, 13, 14], are also often applied to sub-cellular dynamics. In 2005, a model designed to allow the simulation of large numbers of cells, but still taking in consideration cell-shape dynamics with the use of sub-cellular particles, was introduced [15]. In this model (named Subcellular Element Model) cells are not confined to a lattice, being allowed to occupy any position on space. The interaction relations between the sub-cellular units is pre-defined and the interacting forces are calculated using overdamped Langevin dynamics. An interesting application in cell rheology of this model was recently published [16].

An alternative approach of including the cells internal structure in a model to describe cell shape and properties is given in $[17,18]$. In this approach a cell is approximated by a tensegrity structure.

Each of these models described has a specific range of applicability. Factors to take in consideration in order to choose which model to use are, for example, the properties of interest, the size of the system, the need to use physical interactions and the degree of details needed in the internal structure.

The model presented here embraces the strong features of the aforementioned models into a single modeling framework. It is an off-lattice agent-based model and can be run with any subcellular resolution. Many particle interactions are used to define a cell. Neighborhood relations are defined via a Delaunay triangulation and the interacting particles have the possibility to hold specific functions, like being part of the cell membrane or represent an organelle. It is suitable to simulate single cells, a few interacting cells or multi-cellular systems.

The use of Delaunay triangulations allows a faster update of the neighborhood relations between the interacting particles in relation to traditional off-lattice methods. Some methods from molecular dynamics (like the one used in $[15,16]$ ) also provide efficient procedures to update neighborhood relations. Apart from the method used in the definition of neighborhood relations, the model presented in this article and the Subcellular Element Model have similar properties.

Delaunay triangulations have often been used in Biology, usually in the simulation of systems where many cells interact with each other (for example tissues, primary lymphoid follicles formation) $[11,12]$. The model described here is an extension on a framework developed in the last years $[19,20]$. The program generates kinetic (moving vertices) and dynamic (changing number of vertices) Delaunay triangulations for a set of agents, being particularly useful for the simulation of evolving biological systems, where the number of agents varies and at every time step the neighborhood relations must be adjusted.

This framework was extended by adding the possibility that particles interact only in a subset of the defined interaction relations, more specifically in a subset of the Delaunay triangulation restricted to a two-dimensional surface embedded in three-dimensional space. The model will be applied to define the interaction relations between membrane sub-particles of a three dimensional cell. The details of the mathematical implementation will be discussed in Section 3. 


\section{Delaunay triangulation}

\subsection{The Voronoi tesselation}

For every point $p$ in a sample $P$, a Voronoi cell $V_{p}$ can be defined as all points $x$ in $\mathbb{R}^{3}$ that are nearer to this point than to any other point $p^{\prime}$ in the sample. In mathematical terms:

$$
V_{p}=\left\{x \in \mathbb{R}^{3}:|x-p|<\left|x-p^{\prime}\right|, \forall p^{\prime} \in P, p^{\prime} \neq p\right\} .
$$

A Voronoi diagram (or Voronoi tessellation) is the set of Voronoi cells for all points of the sample $P$, and decomposes the $\mathbb{R}^{3}$ into convex polyhedrons (see figure 1 ).

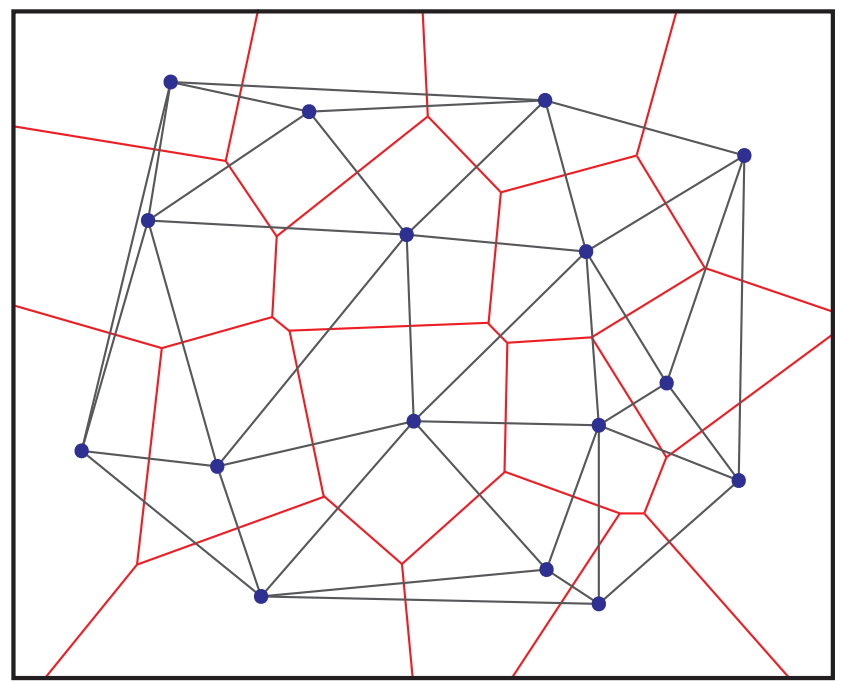

Figure 1: Voronoi tesselation and Delaunay triangulation of a sample $P$ of points in twodimensions. The round dots are the points in the sample $P$, with the connections between them forming their Delaunay triangulation. All other drawn connections belong to the Voronoi tesselation of the sample. In the limit of single vertices representing single cells, Voronoi cells can be associated with biological cell shapes. In this case, the connections on the dual Delaunay triangulation (see section 3.2.) would give the neighborhood relations between cells.

\subsection{Defining the Delaunay triangulation}

Mathematicaly, the Delaunay triangulation (see figure 1) of $P$ is the dual graph of its Voronoi tessellation. Starting with the Voronoi diagram for a sample $P$, the Delaunay triangulation of the sample can be intuitively constructed by connecting every pair of points in $P$ whose Voronoi cells have a common facet. 
A simplex is defined as the convex hull of four points in $P$ whose corresponding Voronoi cells intersection is non-empty. Every point in $P$ is a Delaunay vertex. Throughout this work the terms vertex and point will refer to Delaunay vertices. Edges belonging to the Delaunay triangulation will be referred as connections.

A more formal definition of a Delaunay triangulation is the following: a simplex whose vertices are the points $p_{1}, p_{2}, p_{3}, p_{4}$ belongs to the Delaunay triangulation if the intersection of every pair of Voronoi cells corresponding to two of these points is non-empty.

\subsubsection{The Delaunay criterion}

When the Voronoi tesselation is not known a priori, the Delaunay criterion is used in order to construct a Delaunay triangulation.

Every simplex of vertices $p_{i}$ in $\mathbb{R}^{3}$ has a circumsphere. The radius and the center of the circumsphere can be derived from the four sphere equations

$$
\left(m-p_{i}\right)^{2}=R^{2}, \quad i=1, \ldots, 4,
$$

where $m$ is the coordinates of the center of the circumsphere and $R$ is its radius.

For a triangulation to be considered a Delaunay triangulation, all of its simplices must satisfy the empty-circumsphere-criterion, i.e., no vertex of the triangulation may lie inside the circumsphere of the triangulation simplices. Therefore, a Delaunay triangulation is uniquely defined if the points in $\mathrm{P}$ are in extended general position, i.e., no two points are identical, no three points lie on a common line, no four points lie on the same plane and no five points lie on a common sphere (for the three-dimensional case discussed here) [19, 21, 22, 23].

To verify whether or not a vertex lies inside the circumsphere of a simplex, the four sphere equations 3.2 can be solved. A more efficient way of solving this problem involves the inclusion of one more dimension. With that, the in-circumsphere-criterion in $\mathbb{R}^{3}$ can be reduced to a orientation computation in $\mathbb{R}^{4}$. More details about this lifting procedure can be found in [21].

\subsubsection{Computational implementation of the three-dimensional Delaunay triangulation}

The underlying algorithms to calculate Delaunay triangulations used in this work have been published before $[19,20,24]$ and are available upon request. The implementation is a fully kinetic and dynamic three-dimensional Delaunay triangulation of a given set of particles, and supports dynamic insertion and deletion of vertices, being therefore specially suitable for defining interaction relations between particles in systems where the number of particles may vary. The implementation was done in $\mathrm{C}++$ and is parallelized $[20,25]$.

\section{Inclusion of subcellular particles}

The Delaunay triangulation has been successfully used to calculate neighborhood relations in simulations of systems involving many cells $[12,26]$. Additionally, its dual graph (the Voronoi tes- 
selation) can approximate quite well the shape of cell in closely packed systems (for example, in tissues [27]).

In order to generalize the simulation framework to the internal structure of a cell, though, the idea of each point of the triangulation representing a single cell must be abandoned. Instead, a cell is to be simulated as a set of interacting sub-particles. Each of the sub-particles will be then represented by a point in the Delaunay triangulation. These sub-particles need not to be all equal; they can have specific properties depending on which part of the cell they belong to. Particles having distinct properties are distinguished by their interaction potentials. Another way to distinguish between different properties is changing the interaction relations between the particles.

\subsection{Distinguishing subcellular particles}

In principle, any kind of internal structure belonging to a cell can be included in the model, by controlling the properties of the sub-particles. This allows, for example, the definition of sub-particles as nucleus or organelles inside the cell, or as belonging to the actin network, the cytoskeleton and any other sub-cellular structure one can think of. However, the more detailed the internal structure of the cell, the more time consuming the computation is, and the inclusion of many of these sub-cellular structures is often not needed.

In the present setup of the model only two different groups of particles are considered: the ones belonging to the membrane and the ones belonging to the internal structure of the cell. Particles belonging to the internal structure interact using the three-dimensional Delaunay triangulation. The particles interact via the Lennard-Jones potential:

$$
U(r)=4 \epsilon\left[\left(\frac{\sigma}{r}\right)^{12}-\left(\frac{\sigma}{r}\right)^{6}\right],
$$

where $\epsilon$ is the depth of the potential and $\sigma$ is the equilibrium distance where the potential is zero. Here $\epsilon=\epsilon^{*} \epsilon_{0}$, where $\epsilon_{0}$ is the universal energy scale, $\sigma=\sigma^{*} \sigma_{0}$ and $r=r^{*} \sigma_{0}$, where $\sigma_{0}$ is the universal length scale. From now on reduced units in terms of $\epsilon_{0}$ and $\sigma_{0}$ will be used. In this case, equation 4.1. becomes

$$
U\left(r^{*}\right)=4 \epsilon^{*}\left[\left(\frac{\sigma^{*}}{r^{*}}\right)^{12}-\left(\frac{\sigma^{*}}{r^{*}}\right)^{6}\right] .
$$

For convenience we drop the stars in the remainder of the paper.

The same is used for the interaction between membrane and internal particles: they follow the neighborhood relations coming from the Delaunay triangulation and the potential is also LennardJones, even though the parameters $\sigma$ and $\epsilon$ differ.

\subsection{Membrane sub-particle interactions}

A cell membrane is mainly composed by a lipid bilayer - where little or no long range interactions happen - and, therefore, can be approximated by a two-dimensional bended surface. Consequently, the interactions between particles used to simulate the membrane should be local. However, albeit 
local, the neighborhood relations coming from the Delaunay triangulation are not sufficiently restrictive to be used here. The connections obtained will still allow interactions to happen outside the two-dimensional surface that defines the cell membrane.

The solution for that is to consider only a subset of the Delaunay triangulation when defining the interaction relations between membrane particles. In this case, only the connections belonging to the Delaunay triangulation that are restricted to the membrane two-dimensional surface should be considered. An overview on how to find the restricted triangulation will be given in subsection 4.2.1.

The interaction potential used for the membrane particle is a simple elastic potential:

$$
U(r)=\frac{1}{2} \kappa\left(r-r_{0}\right)^{2},
$$

where $\kappa$ is the elastic constant and $r_{0}$ is the equilibrium distance of the potential. As before, $\kappa$ and $r_{0}$ are given in reduced units.

\subsubsection{Neighborhood relations between membrane sub-particles}

The problem of finding the neighborhood relations of the membrane particles restricted to the two-dimensional surface has many parallels with problems in surface reconstruction [23, 28, 29]. Starting with a set of points known to belong to a determined surface, how can one reconstruct the original surface? However, the traditional solutions from the area of surface reconstruction cannot be directly applied to the problem discussed here.

The main difference stems from the goals of both problems: in surface reconstruction, the points drawn are just the means to recover the original surface. They can be moved or even deleted, if necessary, during the process of finding the desired surface [28]. In the case of the neighborhood relations between membrane particles, however, its not possible to have membrane points moved or deleted, and the goal is to get a triangulation between these points that lies on a surface, even though there is no original surface to be recovered.

Therefore, although the algorithm used borrows some ideas from surface reconstruction techniques, it was particularly developed to treat the case of neighborhood relations between particles that form a surface, where none of the particles can be moved or deleted.

The algorithm uses as starting point the three-dimensional Delaunay triangulation involving both internal and membrane sub-particles. From this triangulation, only the subset of connections between membrane sub-particles is considered. Since the cell is full of internal particles, the collection of the membrane particles' connections will resemble the surface to be obtained, even though it will still have many connections that do not belong to the restricted triangulation on the surface. That rough surface will be called crust, in reference to a similar procedure from surface reconstruction methods $[28,29]$. A visualization of a part of a crust is given in figure 2.

The following step is to refine the crust, cutting out some of the connections so that the ones left make a proper surface triangulation with the membrane points. There are many possible final configurations that satisfy this criterium, and it will be considered that any solution found is a good one. 


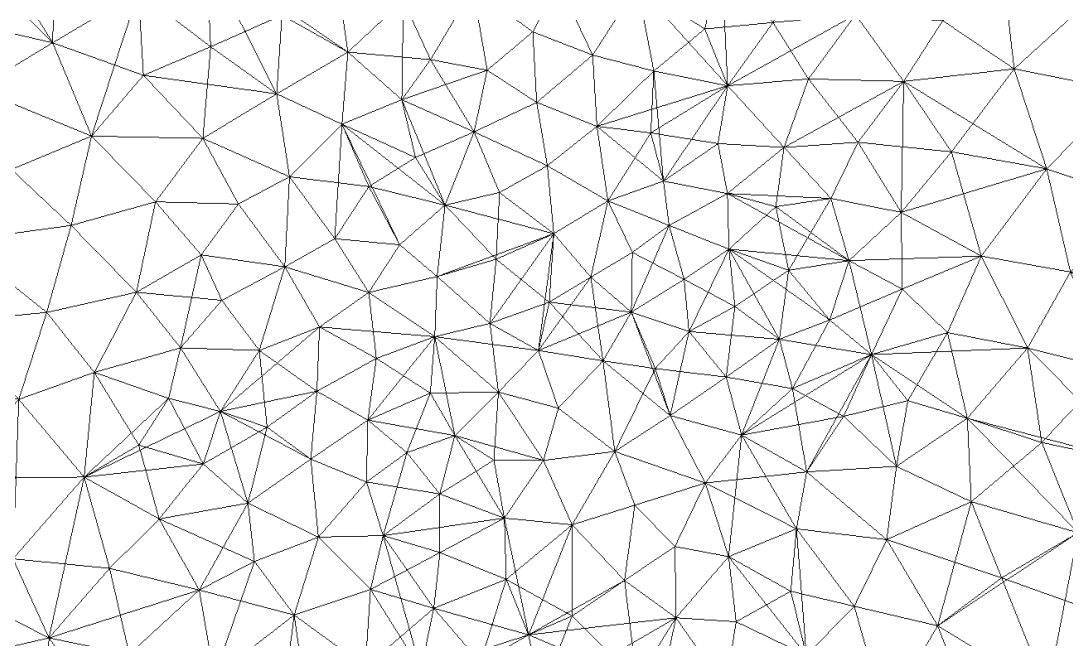

Figure 2: A crust is obtained from a Delaunay triangulation of membrane and internal subparticles, when only the connections between membrane sub-particles are kept. Notice that it is not yet a two-dimensional triangulation of a surface, since it still contains three-dimensional structures (that can be seen here as the structures formed by crossing connections).

The crust will be separated in clusters. Clusters are defined as areas on the crust that are not yet properly triangulated on a two-dimensional surface. Such clusters can be defined because they are surrounded by already two-dimensional surface (see figure 3). Each cluster is then solved independently.

The objective of removing all three-dimensional structures from the clusters is achieved via an iterative procedure. This procedure differ depending on whether the cluster to be solved has or not points that do not belong to its boundary [30].

\subsection{System dynamics}

The evolution of the system in time is calculated using the Verlet algorithm [31, 32]. The position of a particle in the system in a instant of time $t_{0}+\Delta t$ is given by:

$$
x\left(t_{0}+\Delta t\right)=2 x t_{0}-x\left(t_{0}-\Delta t\right)+a \Delta t^{2},
$$

where $a$ is the acceleration of the particle, taken simply from the resulting force acting on it.

Additionally, an adaptive timestep is used. The timestep $(\Delta t)$ is chosen to be the biggest possible so that the displacement of any particle is not bigger than a determined threshold. 


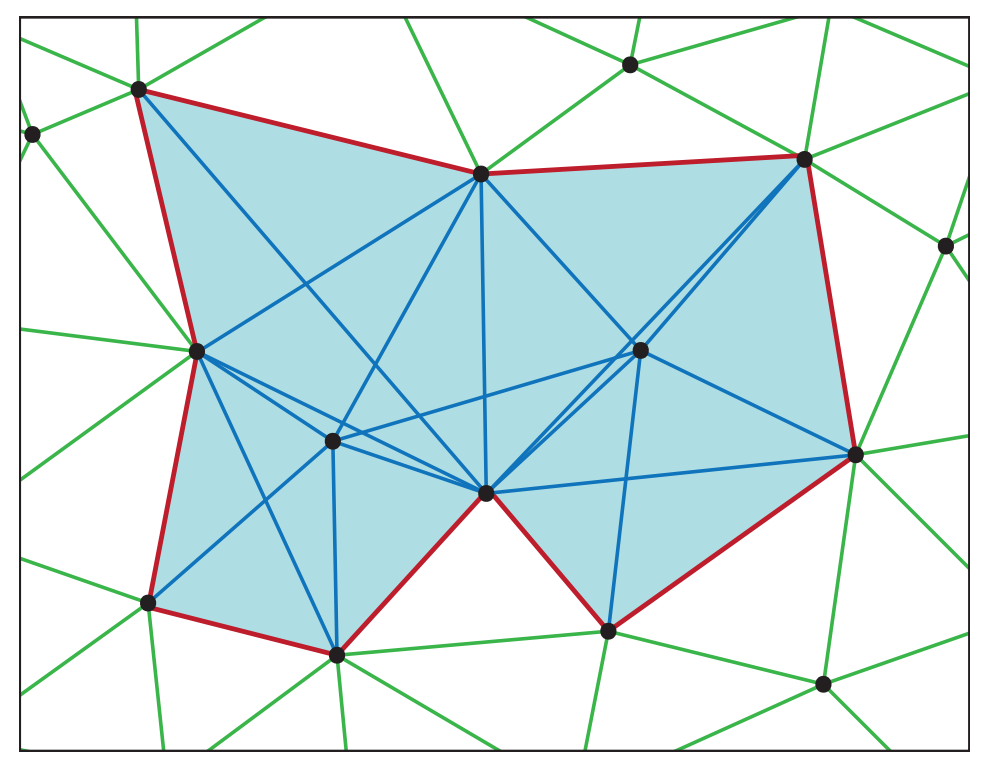

Figure 3: Visualization of a cluster. Note the crossing connections in the highlighted area. This area (i.e., the cluster) is not properly triangulated on a two-dimensional surface, since it still contains simplices (that are, by definition, three-dimensional structures). The surrounding of the cluster already forms a two-dimensional surface.

\section{Example: generating the initial configuration of a cell}

As an example, the thermalization of a cell until its initial condition prior to a simulation is shown. The cell is formed by membrane and internal particles. Membrane particles are initialized randomly on a spherical shell. A single artificial internal particle is put to the center of the sphere. This internal particle interacts with the membrane particles attractively, like a central potential. The membrane particles are confined to the spherical shell by this interaction plus a hard sphere they cannot penetrate into. Within the spherical shell, membrane particles interact elastically. The membrane particles interact without any internal particles but the artificial one responsible for the central force, until they are uniformly distributed on the spherical shell. The configuration is accepted as thermalized after a defined accuracy of the distance between the particles is achieved.

The Delaunay triangulation of all particles involved on the interactions is calculated and used in order to obtain the neighborhood relations of the system. The triangulation is updated at every timestep accordingly to the changes in the particles' positions.

Once the membrane particles are thermalized on the spherical shell, the artificial internal particle is removed and the real internal particles are randomly placed inside the shell. They interact with each other and with the membrane particles using a Lennard-Jones potential (equation 4.1.). However, the membrane particles, at this stage, are fixed and therefore dont feel the interaction with the internal particles. The internal particles then interact until they are thermalized. 


\subsection{Thermalizing membrane particles}

Supposing a cell with $N_{\text {memb }}=1000$ membrane particles, and a rest distance between the particles equal to 1 , the radius $R$ of the spherical shell can be calculated as

$$
R=\sqrt{\frac{\sqrt{3}}{2} \frac{N_{m e m b}}{4 \pi}} .
$$

The elastic constant used on the elastic interaction between membrane points is $\kappa=30$. Since the variance for the average distance between two particles tends to an asymptotic value between $10 \%$ and $15 \%$, thermalization is defined to be achieved when the variance on the average distance is smaller than $14.5 \%$. In figure 4 the membrane particles are shown before and after thermalization.
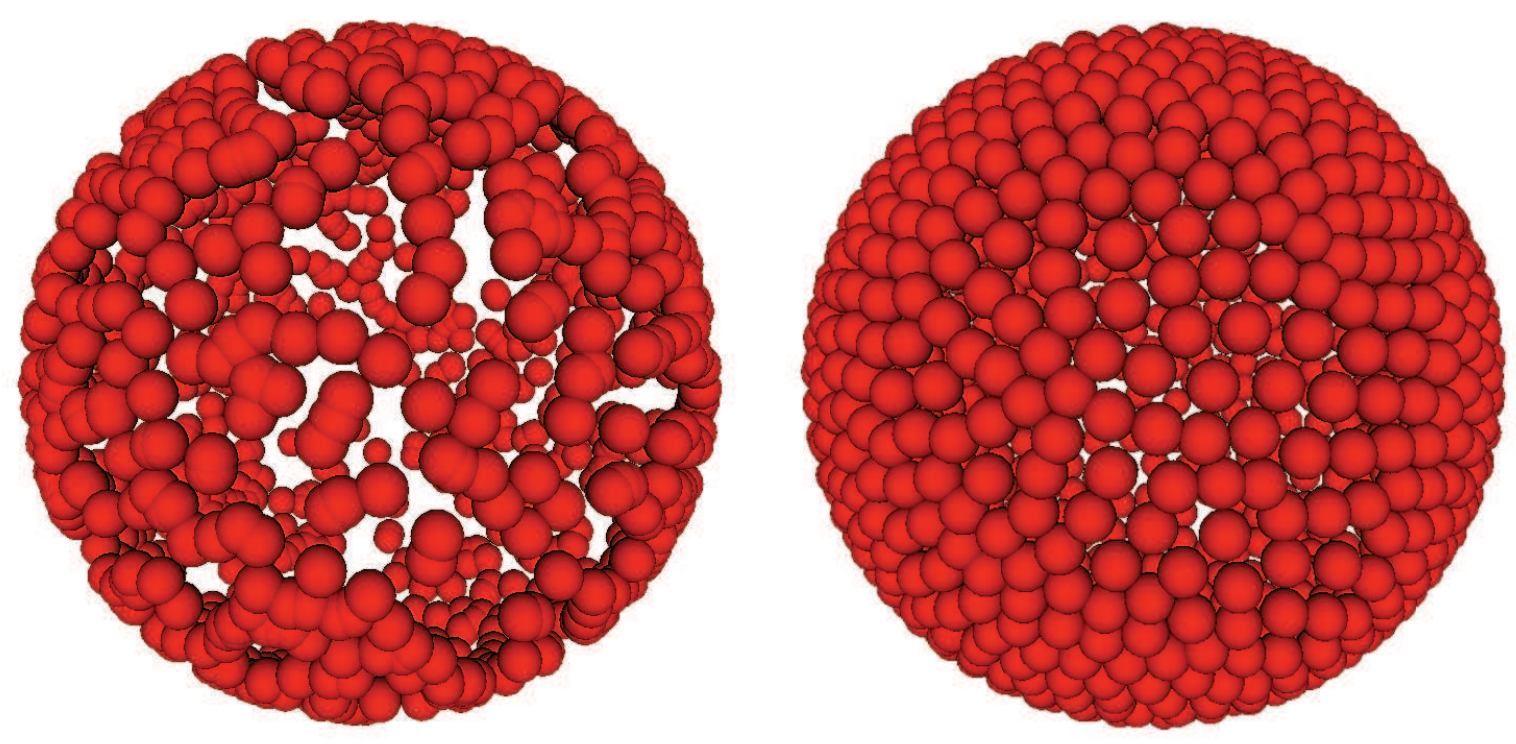

Figure 4: Configuration of membrane particles before (left) and after (right) thermalization.

\subsection{Thermalizing internal particles}

In this example, internal particles are set to have a radius (and, therefore, an interaction rest distance) twice as big as membrane particles. The number of internal particles is calculated by

$$
N_{i n t}=0.74 \frac{\left(R-r_{m e m b}\right)^{3}}{r_{i n t}^{3}},
$$

rounded up to the next integer. Here $r_{m e m b}$ is the radius of a membrane particle and $r_{\text {int }}$ is the radius of an internal particle. The factor 0.74 comes from the supposition that, after thermalization, the 
spheres will be approximately in the densest possible packing (known as cubic close packing). It's been proved that [33], in this case, the density of spheres will be given by

$$
\frac{\pi}{3 \sqrt{2}} \approx 0.74
$$

The set of parameters used for the Lennard-Jones interaction between internal particles was $\epsilon=1$ and $\sigma=2 r_{i n t} 2^{-1 / 6}$, where $2 r_{\text {int }}$ equals the rest distance between the particles. For the Lennard-Jones interaction between membrane and internal particles the parameters were $\epsilon=1$ and $\sigma=\left(r_{\text {int }}+r_{\text {memb }}\right) 2^{-1 / 6}$.

The criteria to define whether thermalization was achieved is the same as for membrane particles. In figure 5, a final thermalized configuration is shown. This configuration is then used as the initial configuration for a cell in a simulation. Additionally, figure 6 shows the visualization of all connections between internal particles (i.e., of the Delaunay triangulation) after thermalization.
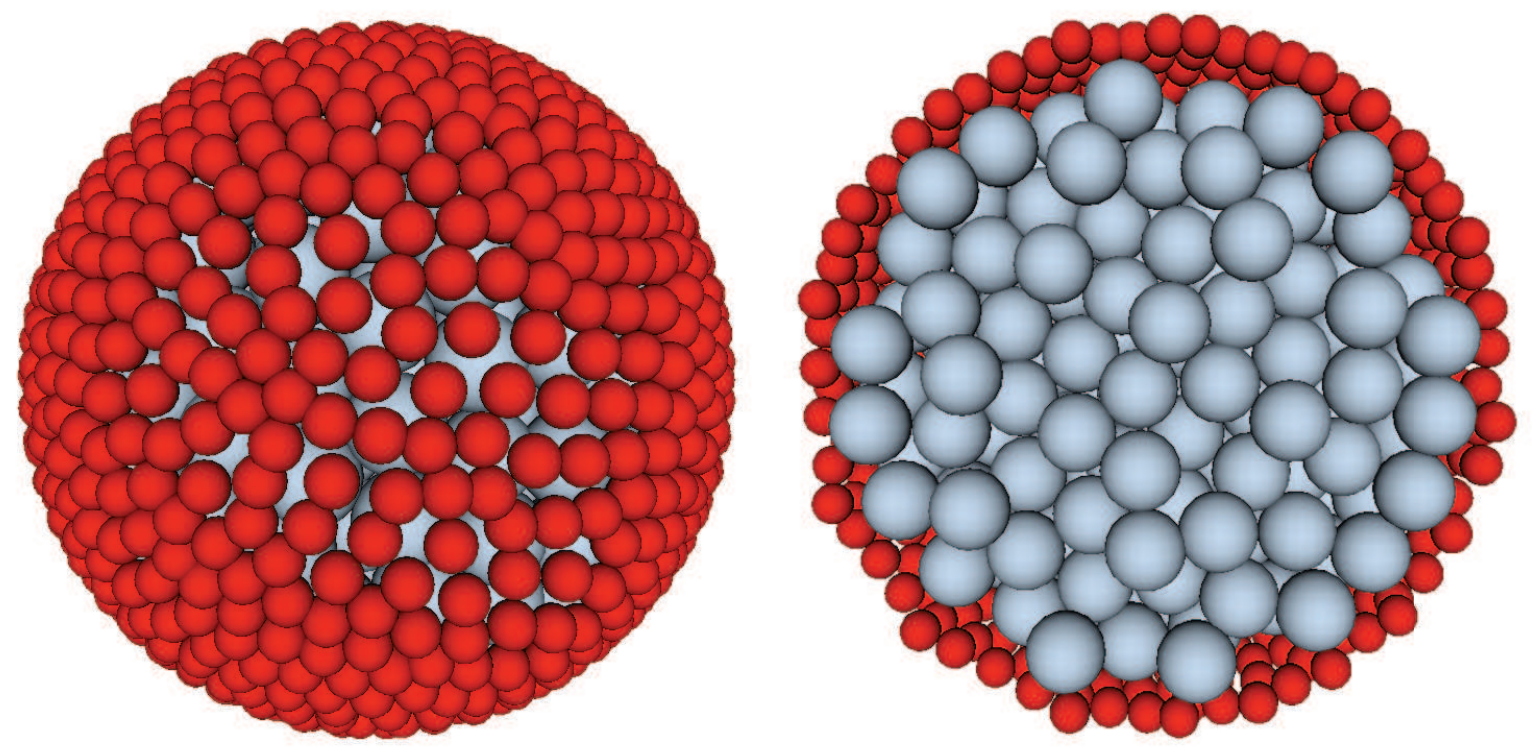

Figure 5: Thermalized configuration with membrane particles (dark colored) and internal particles (light colored). In the right side, the cell is shown sliced in half. Notice that in this example the radius of the internal particles is twice as big as the radius of the membrane particles. The size of the particles is related to the strength of the potential as well as with the resolution used to describe the cell.

\section{Conclusion}

A novel model framework suitable to describe cell shape and behavior was described. The model simulates a cell as a set of discrete particles in continuum space, and allows the introduction of 


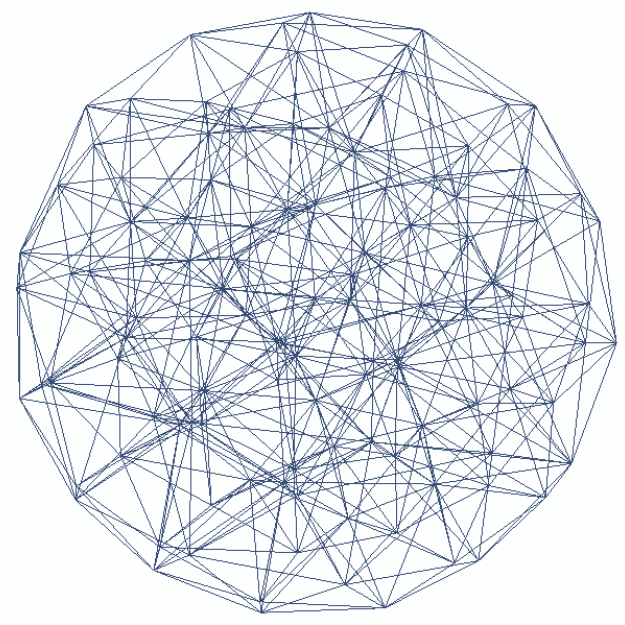

Figure 6: The three-dimensional Delaunay triangulation of all cell internal particles after thermalization, for a simulated system with 500 membrane particles (not shown) and 115 internal particles.

practically any resolution needed for the cell's internal structure. The framework also provides the possibility to include into the model of all kinds of complex structures that are part of the internal structure of a cell, like a nucleus, organelles or the cytoskeleton.

By adjusting the complexity of the cell's internal structure and the number of cells, the method is able to simulate from highly detailed single cells to multi-cellular systems involving an arbitrary number of cells.

Possible mechanical applications involve the simulation of cell migration and its consequential shape changes. On the subcellular level, it becomes more and more evident that it is not only important to have a concentration of molecules in the cell, but also it is needed to have them at the right places. This method is also suitable for the simulation of space-resolved diffusion and transport processes inside a cell, as well as the connection between these molecular processes with cell shape and migration.

\section{Ackowledgements}

The authors thank the ALTANA AG, the EU-NEST project MAMOCELL, and the Vereinigung von Freunden und Förderern der Johann Wolfgang Goethe-Universität Frankfurt am Main e.V. for financial support. 


\section{References}

[1] M. J. Miller, S. H. Wei, I. Parker, M. D. Cahalan. Two photon imaging of lymphocyte motility and antigen response in intact lymph node. Science 296 (2002), 1869-1873.

[2] S. Stoll, J. Delon,T. M. Brotz, R. N. Germain. Dynamic Imaging of T Cell-Dendritic Cell Interactions in Lymph Nodes. Science 296 (2002), 1873-1876.

[3] U. H. von Andrian. T cell activation in six dimensions. Science 296 (2002), 1815-1817.

[4] R. F. Murphy. Putting proteins on the map. Nat. Biotechnol. 24 (2006), 1223-1224.

[5] W. Schubert et al. Analyzing proteome topology and function by automated multidimensional fluorescence microscopy. Nat. Biotechnol. 24 (2006), 1270-1278.

[6] W. Alt, R. T. Tranquillo. Basic morphogenetic system modeling shape changes of migrating cells: how to explain fluctuating lamellipodial dynamics. Journal of Biol. Systems 3 (1995), No. 4 905-916.

[7] M. T. Figge, A. Garin, M. Gunzer, M. Kosco-Vilbois, K.-M. Toellner, M. Meyer-Hermann. Deriving a germinal center lymphocyte migration model from two-photon data. Journal of Exp. Med. 205 (2008), No. 13, 3019-3029.

[8] M. Meyer-Hermann, M. T. Figge, K.-M. Toellner. Germinal centres seen through the mathematical eye: B cell models on the catwalk. Trends in Immunology 30 (2009), No. 4, 157-164.

[9] F. Graner, J. A. Glazier. Simulation of biological cell sorting using a two-dimensional extended Potts model. Phys. Rev. Lett. 69 (1992), No. 13, 2013-2016.

[10] M. E. Meyer-Hermann, P. K. Maini. Interpreting two-photon imaging data of lymphocyte motility. Phys. Review E 71 (2005), No. 6, 061912-061923.

[11] F. A. Meineke, C. S. Potten, M. Loeffler. Cell migration and organization in the intestinal crypt using a lattice-free model. Cell Prolif. 34 (2001), No. 4, 253-266.

[12] T. Beyer, M. Meyer-Hermann. Mechanisms of organogenesis of primary lymphoid follicles. Int. Immunol. 20 (2008), No. 4, 615-623.

[13] M. Bock, A. K. Tyagi, J.-U. Kreft, W. Alt. Generalized Voronoi tessellation as a model of two-dimensional cell tissue dynamics. arXiv:0901.4469v2 [physics.bio-ph].

[14] J. Galle, M. Hoffmann, G. Aust. From single cells to tissue architecture - a bottom-up approach to modeling the spatio-temporal organisation of complex multi-cellular systems. J. Math. Biol. 58 (2009), 261-283.

[15] T. J. Newman. Modeling multicellular systems using subcellular elements. Mathematical Biosciences and Engineering 2 (2005), No. 3, 611-622. 
[16] S. A. Sandersius, T. J. Newman. Modeling cell rheology with the Subcellular Element Model. Phys. Biol. 5 (2008), No. 1, 015002-015014.

[17] D. E. Ingber. Cellular tensegrity I. Cell structure and hierarchical systems biology. J. Cell Sci. 116 (2003), 1157-1173.

[18] D. E. Ingber. Tensegrity II. How structural networks inuence cellular information-processing networks. J. Cell Sci. 116 (2003), 1397-1408.

[19] G. Schaller, M. Meyer-Hermann. Kinetic and dynamic Delaunay tetrahedralizations in three dimensions. Comput. Phys., Commun. 162 (2004), No. 1, 9-23.

[20] T. Beyer, G. Schaller, A. Deutsch, M. Meyer-Hermann. Parallel dynamic and kinetic regular triangulation in three dimensions. Comput. Phys. Commun. 172 (2005), No. 2, 86-108.

[21] A. Okabe, B. Boots, K. Sugihara, S. N. Chiu. Spatial tessellations: concepts and applications of Voronoi diagrams. Probability and Statistics. John Wiley \& Sons, Inc., New York, 1992.

[22] E. Mücke. A robust implementation for three-dimensional Delaunay triangulations. Internat. J. Comput. Geom. Appl. 2 (1998), No. 8, 255-276.

[23] F. Cazals e J. Giesen. Delaunay triangulation based surface reconstruction: ideas and algorithms. Institut National De Recherche En Informatic et en Automatique Rapport de recherche No. 5393 (2004).

[24] M. Meyer-Hermann. Delaunay-Object-Dynamics: cell mechanics with a 3D kinetic and dynamic weighted Delaunay-triangulation. Curr. Top. Dev. Biol. 81(2008), 373-399.

[25] T. Beyer, M. Meyer-Hermann. The treatment of non-flippable configurations in three dimensional regular triangulations. WSEAS Trans. Syst. 5 (2006), No. 5, 1100-1107.

[26] G. Schaller, M. Meyer-Hermann. Multicellular tumor spheroid in an off-lattice Voronoi/Delaunay cell model. Phys. Rev. E 71 (2005), No. 5, 051910-051925.

[27] G. V. Reddy, M. G. Heisler, D. W. Ehrhardt, E. M. Meyerowitz. Real-time lineage analysis reveals oriented cell divisions associated with morphogenesis at the shoot apex of Arabidopsis thaliana. Development 131 (2004), No. 17, 4225-4237.

[28] N. Amenta, M. Bern, M. Kamvysselis. A new Voronoi-based surface reconstruction algorithm. SIGGRAPH '98: Proceedings of the 25th annual conference on computer graphics and interactive techniques, ACM, New York, 1998.

[29] N. Amenta, M. Bern. Surface reconstruction by Voronoi filtering. Discrete and Computational Geometry 22 (1999), No. 4, 481-504.

[30] G. Grise, M. Meyer-Hermann. Surface reconstruction using Delaunay triangulation for applications in life sciences. Submitted (2009). 
[31] L. Verlet. Computer experiments on classical fluids. I. Thermodynamical properties of Lennard-Jones molecules. Phys. Rev. 159 (1967), No. 1, 98-103.

[32] L. Verlet. Computer experiments on classical fluids. II. Equilibrium correlation functions. Phys. Rev. 165 (1968), No. 1, 201-214.

[33] Wu-Yi Hsiang. On the sphere packing problem and the proof of Kepler's conjecture. Internat. J. Math. 4 (1993), No. 5, 739-831. 